\title{
Rapamycin attenuated podocyte apoptosis via upregulation of nestin in Ang II-induced podocyte injury
}

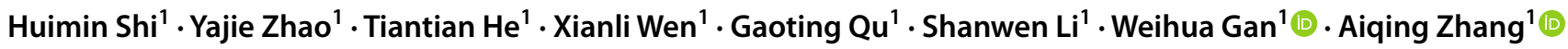

Received: 25 July 2021 / Accepted: 26 November 2021 / Published online: 11 February 2022

(c) The Author(s) 2022

\begin{abstract}
Background Angiotensin II (Ang II) contributes to the progression of glomerulosclerosis, mainly by inducing podocyte injury. Convincing evidence indicates that the mTOR inhibitor rapamycin could play a fundamental role in protection against podocyte injury. Nestin, a major cytoskeletal protein, is stably expressed in podocytes and correlates with podocyte damage. The purpose of this study was to investigate the effect of rapamycin on podocyte injury induced by Ang II and to clarify the role and mechanism of nestin in the protective effect of rapamycin of podocyte injury.

Methods and results We established an Ang II perfusion animal model, and the effects of rapamycin treatment on podocytes were investigated in vivo. In vitro, podocytes were stimulated with Ang II and rapamycin to observe podocyte injury, and nestin-siRNA was transfected to investigate the underlying mechanisms. We observed that Ang II induced podocyte injury both in vivo and in vitro, whereas rapamycin treatment relieved Ang II-induced podocyte injury. We further found that nestin co-localized with p-mTOR in glomeruli, and the protective effect of rapamycin was reduced by nestin-siRNA in podocytes. Moreover, co-IP indicated the interaction between nestin and p-mTOR, and nestin could affect podocyte injury via the mTOR/P70S6K signaling pathway.

Conclusion We demonstrated that rapamycin attenuated podocyte apoptosis via upregulation of nestin expression through the mTOR/P70S6K signaling pathway in an Ang II-induced podocyte injury.
\end{abstract}

Keywords Nestin $\cdot$ Rapamycin $\cdot$ Ang II $\cdot$ Podocyte injury $\cdot$ Apoptosis

\section{Introduction}

Podocytes, which maintain the integrity of the glomerular filtration barrier, are terminally highly differentiated in the renal glomerulus [1]. Podocyte injury causes proteinuria, leading to the progression of glomerular diseases [2]. Angiotensin II (Ang II), an important active effector in the reninangiotensin system (RAS), contributes to podocyte injury, including podocyte apoptosis, cytoskeletal rearrangement, and loss of the slit diaphragm [3-5]. Several prior studies

Huimin Shi and Yajie Zhao have contributed equally to this work.

Weihua Gan

weihuagan@njmu.edu.cn

Aiqing Zhang

njaiqing@njmu.edu.cn

1 Department of Pediatric Nephrology, The Second Affiliated Hospital of Nanjing Medical University, 262 Zhongshan North Road, Nanjing 210003, Jiangsu Province, China have shown that podocyte injury is closely related to the activation of the mammalian target of rapamycin (mTOR) [6-9], which plays an important role in glomerular disease. Rapamycin, an mTOR inhibitor, has been shown to have immunosuppressive and antiproliferative efficiency $[10,11]$. It has been revealed that rapamycin treatment protected a rat model of diabetic nephropathy from kidney impairment [12].

Nestin is a class VI intermediate filament (IF) protein that was initially characterized in neuroepithelial stem cells [13] and participates in the composition of the cytoskeleton. Nestin is stably expressed in mature podocytes and plays a significant role in maintaining the normal morphology and function of podocytes $[14,15]$. However, the signaling mechanism involved in nestin function in glomerular podocytes has not yet been established.

We hypothesized that rapamycin attenuates podocyte injury under Ang II conditions and that nestin could play an important role in this process. Therefore, in this study, we explored the effects of rapamycin on podocytes by 
investigating the changes in nestin expression and elucidating the underlying mechanisms.

\section{Materials and methods}

\section{Animals}

Eight-week-old, 20-22-g male C57BL/6 mice (Vital River Laboratory Animal Technology, Beijing, China) were included in the experiments. The experimental procedures were approved by the Institutional Animal Care and Use Committee of the Nanjing Medical University. The mice underwent a sham operation under light anesthesia with $3 \%$ isoflurane. Two weeks after surgical intervention, an osmotic minipump (model 2004; Alzet, Cupertino, CA) was implanted subcutaneously in mice to infuse Ang II for 4 weeks. The mice were randomly divided into three experimental groups: control group (sham operation, $\mathrm{n}=6$ ); Ang II group (Ang II, 400 ng/kg/min [16], subcutaneouslyembedded anosmotic minipump, $\mathrm{n}=6$ ); and Ang II + RP group (Ang II, $400 \mathrm{ng} / \mathrm{kg} / \mathrm{min}$, subcutaneously-embedded minipump, intraperitoneal injection $1 \mathrm{mg} / \mathrm{kg} /$ day $\mathrm{RP}, \mathrm{n}=6$ ). The drinking water of all groups was supplemented with $1 \%$ sodium chloride throughout the experimental period. Kidneys were collected for biochemical and renal pathological analyses.

\section{Cell culture}

The immortalized mouse podocyte cell lines (MPCs) used in this study were gifted by Dr. Junwei Yang (Nanjing Medical University). Podocytes were cultured in RPMI-1640 medium (Gibco, USA) with 10\% fetal bovine serum (FBS), $100 \mathrm{U} / \mathrm{ml}$ penicillin G (Gibco, USA), and $10 \mathrm{U} / \mathrm{ml}$ recombinant mouse IFN- $\gamma$ at $33{ }^{\circ} \mathrm{C}$ for proliferation. Podocytes were cultured without IFN- $\gamma$ at $37^{\circ} \mathrm{C}$ for $10-14$ days to induce differentiation and quiescence. The cells were then exposed to Ang II $\left(10^{-6} \mathrm{~mol} / \mathrm{l}\right)$ and rapamycin $(500 \mathrm{ng} / \mathrm{ml})$ treatment for the indicated time periods.

\section{Transfection of small interference RNA}

MPCs were transfected with nestin siRNA (sense, 5'- GGA AGUGACUAGUGAGACATT- ${ }^{\prime}$ ' and antisense, 5'- UGU CUCACUAGUCACUUCCTT-3') (GeneChem, Shanghai, China) using Lipofectamine 2000 transfection reagent (Invitrogen, USA), according to the manufacturer's instructions. In brief, nestin siRNA was diluted into each 6-well plate at a concentration of $40 \mathrm{nM}$ with transfection medium (Opti-MEM, Invitrogen, USA) and incubated for $5 \mathrm{~min}$. Diluted lipofectamine reagent with Opti-MEM and siRNA was mixed and incubated at room temperature for $20 \mathrm{~min}$.
After $6 \mathrm{~h}$ of transfection, the cells were used for further experiments.

\section{Immunohistochemistry}

Immunohistochemical staining was performed on $4 \%$ paraformaldehyde-fixed, paraffin-embedded $3-\mu \mathrm{m}$ renal tissue sections. After antigen recovery, the sections were incubated with primary antibodies against nephrin (1:200, mouse, sc-377246) overnight at $4{ }^{\circ} \mathrm{C}$, and then incubated with peroxidase-conjugated anti-mouse IgG antibodies. Reactions were stained with a DAB substrate kit (MXB biotechnologies, DAB-0031, Fuzhou, China), and counterstaining was performed using hematoxylin. The sections were captured under a microscope (Olympus, BX53, Tokyo, Japan), and Image ProPlus was used to quantify the average optical density value.

\section{Immunofluorescence staining}

Kidney paraffin sections ( $3 \mu \mathrm{m}$ thick) were prepared. Cells grown on glass cover slips were fixed with $4 \%$ paraformaldehyde for $15 \mathrm{~min}$ and permeabilized with $0.1 \%$ Triton X-100 for $5 \mathrm{~min}$ at room temperature. After blocking with $5 \%$ BSA for $1 \mathrm{~h}$, the kidney and cell slides were incubated with primary antibodies against WT1(1:200, rabbit, \#83,535, Cell Signaling Technology), p-mTOR (1:100, rabbit, \#5536, Cell Signaling Technology), nestin (1:200, mouse, sc-23927, Santa Cruz Biotechnology), and nephrin (1:200, mouse, sc-376522, Santa Cruz Biotechnology) in PBS containing $1 \% \mathrm{BSA}$ at $4{ }^{\circ} \mathrm{C}$ overnight. FITC/TRITCconjugated IgG was used as a secondary antibody for $1 \mathrm{~h}$ at room temperature. All samples were mounted with DAPI dye for $5 \mathrm{~min}$ and captured under a confocal microscope (Olympus FV1000, Tokyo, Japan).

\section{Annexin V staining assay}

Podocytes from different groups were quantified by annexin $\mathrm{V}$ staining according to the manufacturer's instructions (KeyGEN Biotech, Jiangsu, China). Podocytes were harvested and washed twice with phosphate-buffered saline (PBS). The cells were resuspended in $100 \mu \mathrm{l}$ of ice-cold binding buffer and incubated with $5 \mu$ of Annexin V (conjugated with FITC) for $15 \mathrm{~min}$ in the dark. After resuspension in $400 \mu \mathrm{l}$ of binding buffer, the cells were observed under a fluorescence microscope (Olympus, BX53, Tokyo, Japan).

\section{TUNEL assay}

Tissue sections ( $3 \mu \mathrm{m})$ were used to detect DNA fragmentation for apoptosis using the One Step TUNEL Apoptosis Assay Kit (KeyGEN Biotech, Jiangsu, China) according to 
the manufacturer's instructions. Kidney cells with TRITC nuclear markers were considered TUNEL-positive. The cells were counterstained with DAPI, and fluorescent images were acquired using a confocal microscope.

\section{Western blotting assay}

Renal tissues and cultured cells were harvested after treatment and lysed in RIPA lysis buffer, supplemented with a protease inhibitor cocktail. The BCA assay (KeyGEN Biotech, Jiangsu, China) was used to measure protein concentration. Protein expression was detected by western blotting, according to established protocols [17]. The same quantities of protein were separated by $10 \%$ SDS-PAGE and transferred to a PVDF membrane (Millipore, HATF09025). The membranes were blocked with 5\% BSA in TBST for $1 \mathrm{~h}$. Membranes were incubated overnight at $4{ }^{\circ} \mathrm{C}$ with the following primary antibodies: nephrin (1:1000, mouse, sc-376522, Santa Cruz Biotechnology), Nestin (1:500, mouse, sc-23927, Santa Cruz Biotechnology), Bax (1:1000, rabbit, AF0120, Affinity Biosciences), p53 (1:1000, mouse, BF8013, Affinity Biosciences), and GAPDH (1:1000, rabbit, \#5174, Cell Signaling Technology) were used. Secondary HRP-conjugated goat anti-mouse or anti-rabbit antibodies (Cell Signaling Technology) were used. Western ECL substrate (Biosharp, China) was used to visualize proteins.

\section{CO-immunoprecipitation (CO-IP) and immunoblotting}

Cells were lysed in lysis buffer, and proteins were immunoprecipitated from the cell lysates with the indicated primary antibodies overnight at $4{ }^{\circ} \mathrm{C}$, according to the manufacturer's instructions (abs955, Absin, Shanghai, China). Immunoprecipitates were mixed with protein $\mathrm{G}$ agarose beads for $1 \mathrm{~h}$ at $4{ }^{\circ} \mathrm{C}$, collected, and washed with lysis buffer. After incubation, the immunoprecipitate complexes were rinsed, and the contents of the sample were analyzed by immunoblotting.

\section{Statistical analysis}

The results are expressed as the mean \pm SEM from at least three independent experiments. Statistical analysis was performed using Prism 6.0 for Windows (GraphPad Software, Inc., California, USA). Inter-group comparisons were assessed using ordinary ANOVA followed by Bonferroni's multiple comparison post-hoc test. A two-sided P-value of $<0.05$ indicated statistical significance.

\section{Results}

\section{Rapamycin attenuated podocyte injury in Ang Il-infused mice}

We established an Ang II-infused mouse model to investigate the effects of rapamycin on glomerular and podocyte injury. The dysregulation or loss of nephrin, an important structural molecule in the silt diaphragm, has been suggested to precede podocyte loss. As shown in Fig. 1, the deletion of nephrin was confirmed via immunohistochemistry and western blotting assays of kidney sections after Ang II stimulation compared with that in the control group. In the presence of rapamycin, podocyte injury induced by Ang II was attenuated. A higher expression level of nephrin was observed after injection of rapamycin compared to that in the Ang II group (Fig. 1a and d). Additionally, immunochemical staining for Wilms' tumor protein (WT1), a surrogate marker of podocyte number, suggested that the number of podocytes was decreased in the Ang II group compared with that in the control group. The number of WT-1 immunopositive podocytes was higher in the glomeruli of the rapamycin-treated group than in glomeruli of the Ang II group (Fig. 1b). The TUNEL assay showed that Ang II induced glomerular apoptosis, and rapamycin treatment could reduce apoptosis (Fig. 1c). The expression of renal apoptosis-related proteins, including Bax and p53, was measured to investigate the effects of rapamycin on renal apoptosis. In the Ang II group, remarkable upregulation of Bax and p53 expression was observed, whereas significant inhibition of Bax and p53 expression was observed after rapamycin treatment (Fig. 1d). These results suggest that rapamycin attenuates podocyte injury in Ang II-infused mice.

\section{Inhibition of mTOR signaling activation by rapamycin restored nestin expression in the glomeruli of Ang II-infused mice}

We evaluated the expression level of signal transduction in renal tissue samples using p-mTOR immunofluorescence. As demonstrated in Fig. 2, the expression of p-mTOR in glomeruli was increased significantly, suggesting activation of mTOR signaling. Further, the Ang II + RP group exhibited lower p-mTOR expression than Ang II group. Interestingly, nestin, a cytoskeleton disruption, was colocalized with p-mTOR in the glomeruli. Nestin expression was weakly observed in the glomeruli of the Ang II group. In the rapamycin-treated group, nestin expression was higher. These results indicated that rapamycin attenuated mTOR signaling activation by restoring nestin expression in the glomeruli of Ang II-infused mice. 


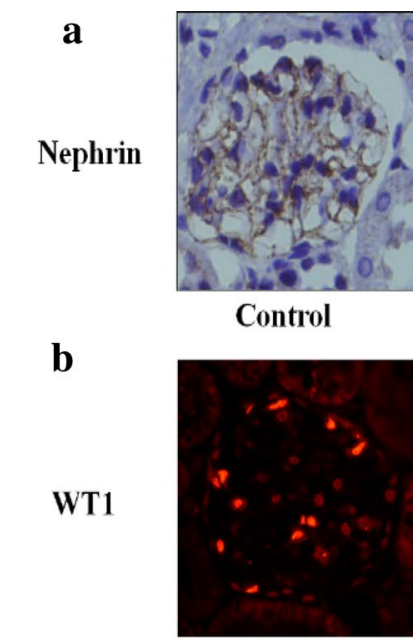

Control

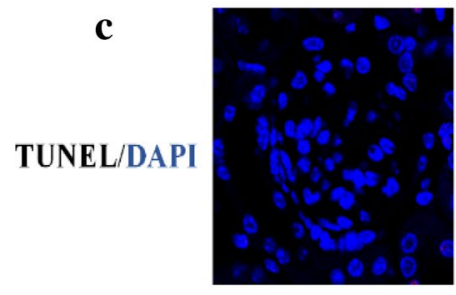

Control

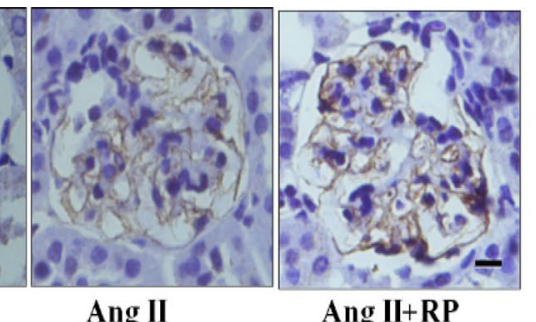

Ang II

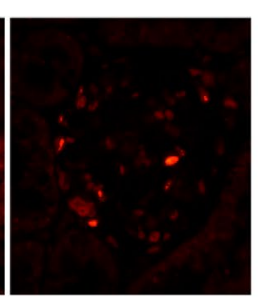

Ang II

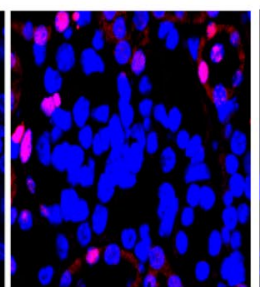

Ang II

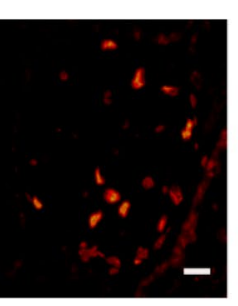

Ang II+RP

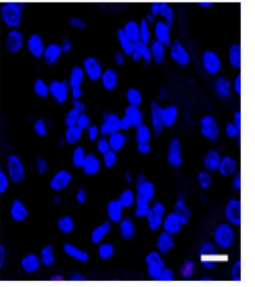

Ang II+RP
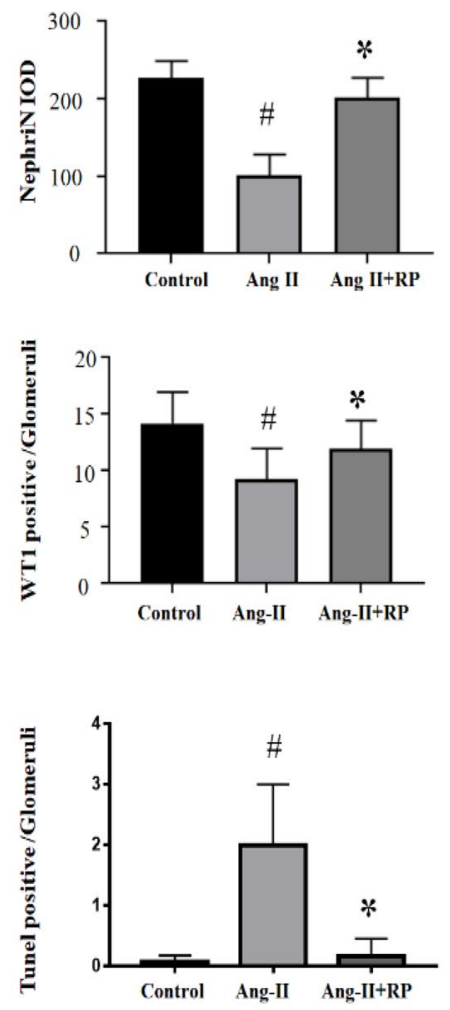

d

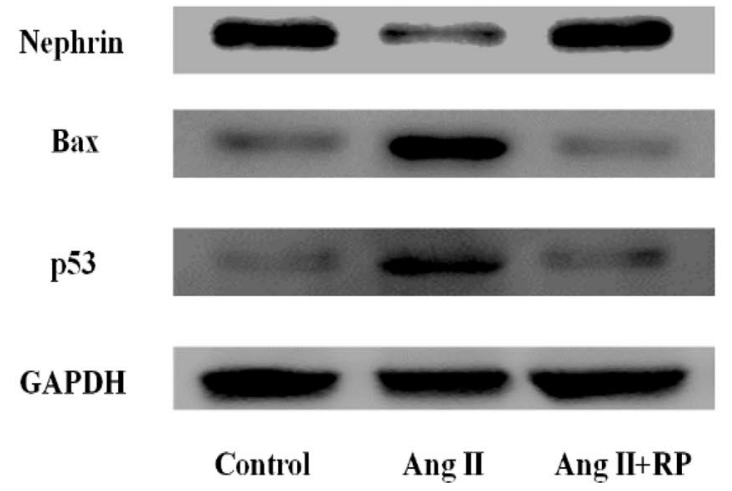

Fig. 1 Rapamycin attenuated podocyte injury in Ang II-infused mice. a As indicated, Immunohistochemical staining for nephrin in kidney tissue from each group. Magnification, $\times 400$. b Representative images of WT-1 immunofluorescent staining in kidney tissues from the above group. Magnification, $\times 400$. c TUNEL staining (Red) in kidney sections after the indicated treatment. The sections were coun-

\section{Inhibition of nestin attenuated the protective effect of rapamycin against Ang II-induced podocyte injury}

To further explore the effect of nestin on the protection from rapamycin, podocytes were cultured and differentiated as previously described. We employed siRNA interference to

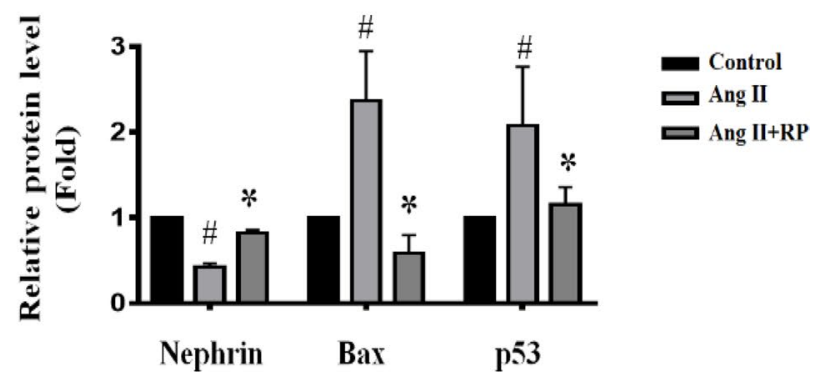

terstained with DAPI (Blue). Magnification, $\times 400$. d Western blotting analysis of nephrin, Bax and p53 expression in the kidney. The levels of GAPDH were used as standard loading controls. The data are presented as mean \pm SEM. $\# \mathrm{P}<0.05$ vs. control group, $* \mathrm{P}<0.05$ vs. Ang II group. (Color figure online)

assess the effect of nestin depletion prior to subjecting podocytes to Ang II stimulation. Nestin expression was decreased in podocytes under Ang II conditions, whereas rapamycin treatment upregulated nestin expression. Following transfection with nestin siRNA, we observed that the morphology of podocytes changed significantly, and that podocyte mutation was short or disappeared by immunofluorescence 

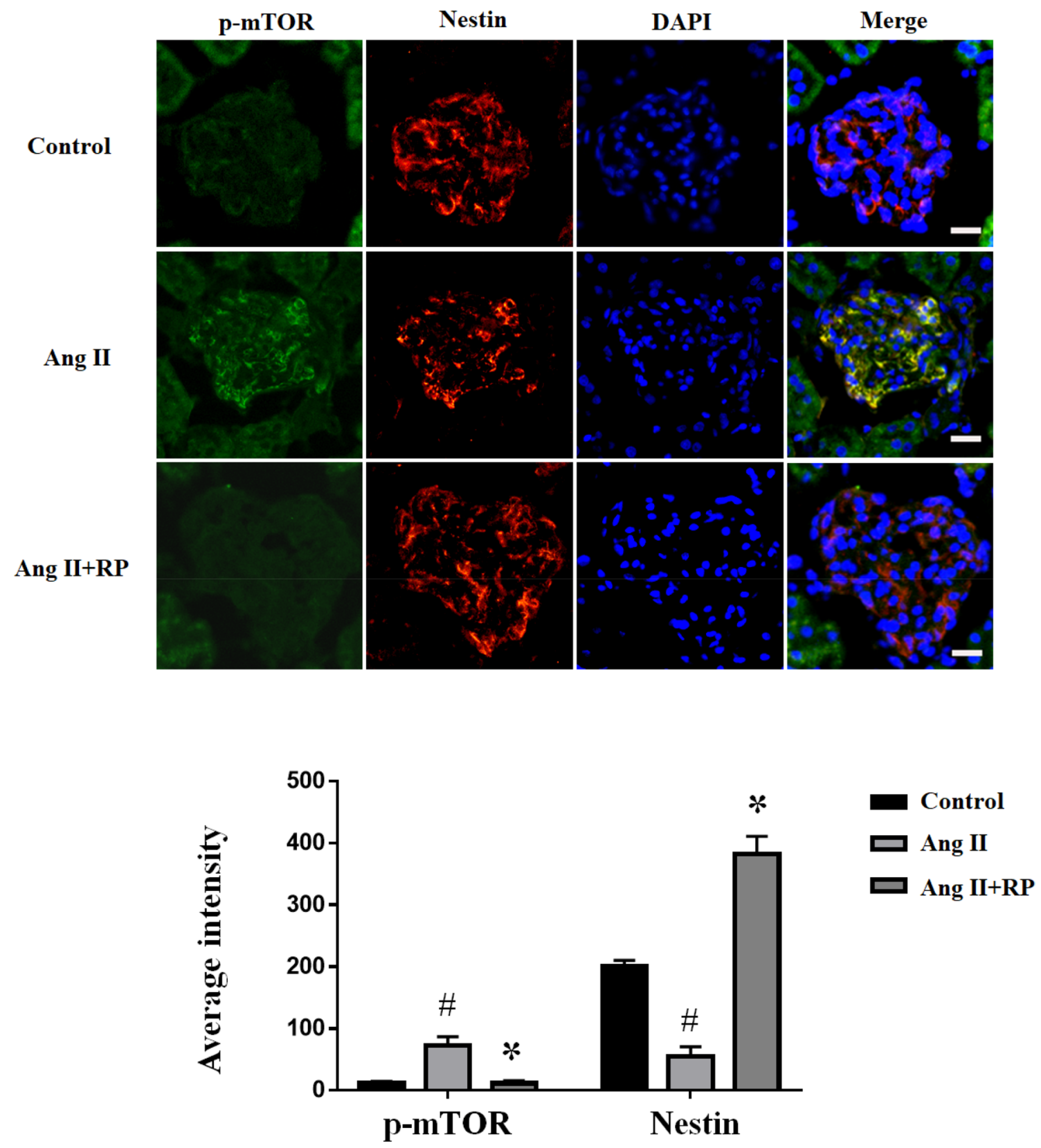

Fig. 2 Inhibition of mTOR signaling activation by rapamycin restored nestin expression in glomeruli of Ang II-infused mice. Indirect immunofluorescent staining showed the expression and localization of p-mTOR (green), nestin (red), and DAPI (blue) in glomeruli from

(Fig. 3a). In addition, Ang II treatment led to a decrease in nephrin expression, and a higher nephrin protein level was detected in the Ang II + RP group compared with in the Ang II group. Meanwhile, nephrin expression was downregulated when nestin-siRNA was used (Fig. 3b). The protein expression of nestin and nephrin was also detected by western blotting, and the results were consistent with the changes observed in immunofluorescence (Fig. 3d). To determine the effect of nestin on podocyte apoptosis, the cells were various group, as indicated. Magnification, $\times 400$. Fluorescence average intensities of p-mTOR and nestin in glomeruli after the indicated treatment. The data are presented as mean \pm SEM. \#P $<0.05$ vs. control group, $* \mathrm{P}<0.05$ vs. Ang II group. (Color figure online)

stained with FITC-Annexin V. Podocyte apoptosis increased significantly when treated with Ang II, as evidenced by the number of Annexin V-positive cells (green), and RP treatment reduced podocyte apoptosis. We found that the number of apoptotic podocytes was enhanced in cells co-treated with nestin siRNA compared with that in the Ang II + RP group (Fig. 3c). We also investigated the expression of Bax and p53. Western blotting analysis revealed that Bax and p53 protein levels were increased under Ang II stimulation and 

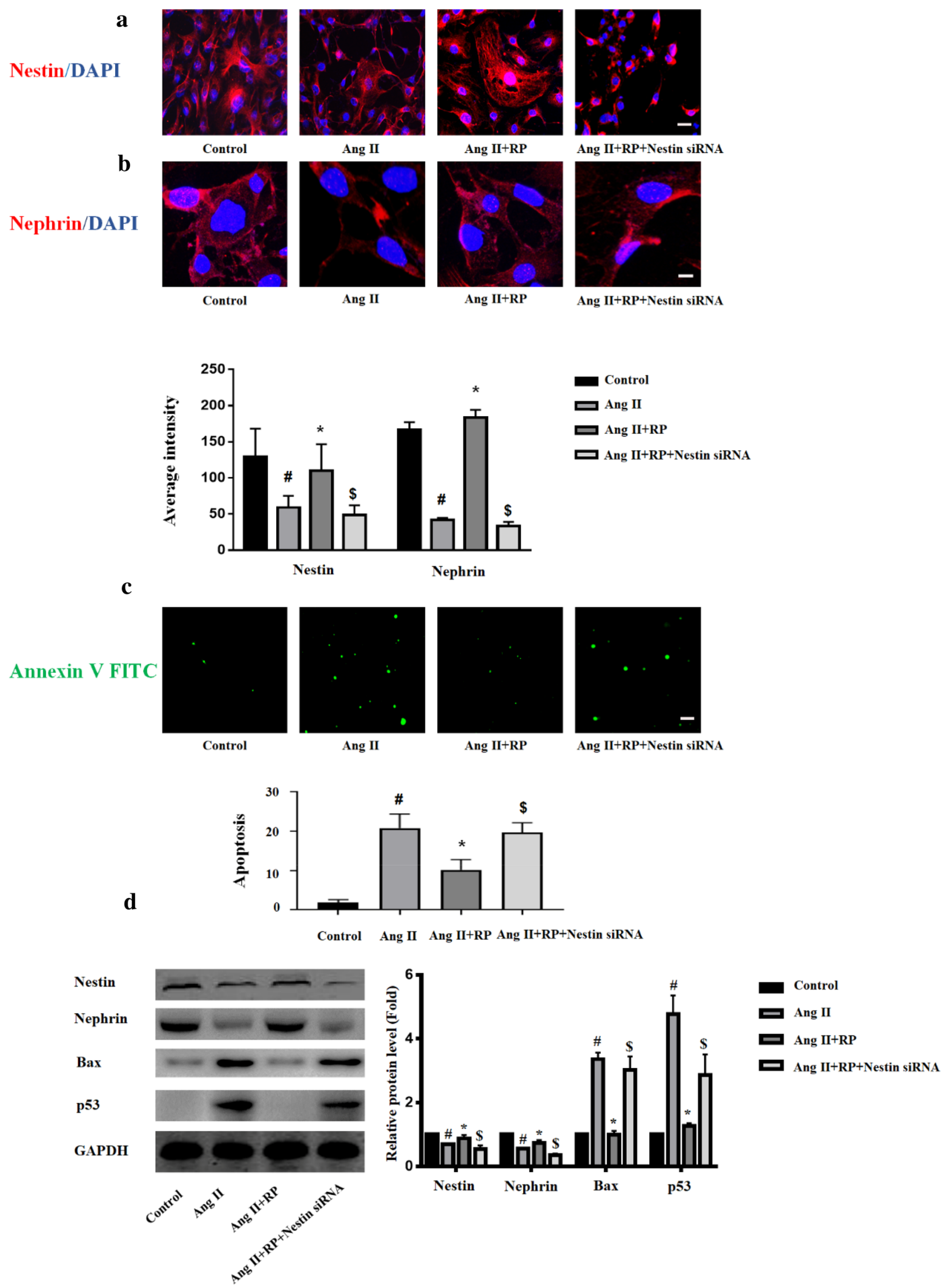
4Fig. 3 Rapamycin in Ang II-induced podocyte injury. a Immunofluorescence staining showed the expression of nestin (Red) in podocyte after the indicated treatment. Magnification, $\times 200$. b Immunofluorescence staining showed the expression of nephrin (Red) in podocyte after the indicated treatment. Magnification, $\times 400$. Fluorescence average intensities of nestin and nephrin in podocytes after the indicated treatment. c Annexin V FITC staining (Green) in podocyte after the indicated treatment. Magnification, $\times 200$. d Western blotting for nestin, nephrin, Bax and p53 after the indicated treatment. The data are presented as mean \pm SEM. $\# \mathrm{P}<0.05$ vs. control group, $* \mathrm{P}<0.05$ vs. Ang II group. $\$ \mathrm{P}<0.05$ vs. Ang II $+\mathrm{RP}$ group. (Color figure online)

decreased when co-treated with Ang II and RP compared to those in the Ang II alone treatment. Corresponding to cellular apoptosis, the expression of Bax and p53 was higher when knocked down with nestin siRNA than in the Ang II + RP group (Fig. 3d).

\section{Nestin combines with p-mTOR, and inhibition of nestin enhances $\mathrm{mTOR} / \mathrm{P70S6 \textrm {K }}$ activation}

Immunofluorescence staining showed that nestin and p-mTOR co-localized in the glomeruli. Co-IP assays were carried out to verify the interaction between nestin and p-mTOR. Co-IP demonstrated that nestin interacted with p-mTOR (Fig. 4a). We then detected the phosphorylation levels of mTOR and P70S6K proteins by western blotting (Fig. 4b), and analyzed the relative levels of mTOR, p-mTOR, P70S6K, and p-P70S6K. The levels of mTOR and P70S6K proteins were approximately equal in each group. Western blotting also revealed a significant increase in the p-mTOR and p-P70S6K protein levels in the Ang II group compared with those in the control group. Furthermore, the levels of p-mTOR and p-P70S6K proteins decreased when treated with rapamycin. Interestingly, compared with that in the Ang II + RP group, mTOR and P70S6K phosphorylation was upregulated when nestin was knocked down.

\section{Discussion}

Several clinical and experimental studies have suggested that podocyte injury is a frequent pathological phenomenon due to various stresses and pathological stimuli, causing proteinuria formation and detachment from the glomerular basement membrane in kidney diseases [1]. Previous studies have confirmed that Ang II, an important active effector in RAS, plays a critical role in CKD progression and podocyte injury [18]. It has been reported that Ang II infusion contributes to proteinuria and hypertension in vivo, subsequently leading to podocyte injury, especially apoptosis [19]. We found that Ang II infusion induced podocyte injury in vivo, followed by a decrease in nephrin expression and loss of podocytes, as revealed in a previous study.
The mTOR pathway, which plays a central role in cellular growth, metabolism, apoptosis, and proliferation, is essential for kidney development during physiological body growth [20]. A growing number of studies have shown that the mTOR signaling pathway is an important regulator of podocyte homeostasis and tubelar transportation in renal diseases [21, 22]. In podocytes, abnormally integrated mTOR signals can be activated by numerous growth factors and cytokines. Excessive activity of mTOR complexes can result in severe pathologic effects, including mislocalization of slit diaphragm proteins, thickening of the glomerular basement membrane, loss of podocytes, and effacement of podocyte foot-press [23]. Rapamycin, an inhibitor of mTOR signaling, has been proven to be an effective therapeutic approach in animal models of glomerular disease and in clinical studies [24]. Our data showed that mTOR signaling was activated after stimulation with Ang II in vivo, and podocyte damage was involved in the activation of the mTOR pathway. In addition, we found that rapamycin attenuated podocyte apoptosis in Ang II-induced podocyte injury.

Nestin, a cytoskeleton-associated class VI intermediate filament (IF) protein, is transiently expressed in glomerular endothelial cells and tubular epithelial cells during renal development. In addition, nestin is highly expressed in mature glomerular podocytes [25]. Recent findings indicate that cytoskeleton disruption is related to podocyte injury [26]. It has previously been demonstrated that nestin plays an important role in maintaining the stability of podocytes and normal podocyte function [14]. Further, nestin expression in podocytes is closely related to proteinuria in kidney diseases, and alterations of nestin may occur to enable podocytes to undergo morphological changes [27]. In the present study, decreased nestin expression and increased p-mTOR expression were observed in Ang II-induced glomeruli. In addition, we found that rapamycin inhibited mTOR activation, followed by upregulation of nestin expression. We surmised that nestin plays an important role in the protection of rapamycin in Ang II-induced podocyte injury. To investigate the role of nestin in this process, we employed siRNA interference to assess the effect of nestin depletion prior to podocyte treatment. Our results showed that the restorative effect of rapamycin on nephrin expression was attenuated by nestin inhibition in Ang II-induced podocyte injury. The number of apoptotic podocytes increased when infected with nestin-siRNA. These results suggest that nestin is not only a marker of podocyte injury, but also a damage-promoting factor in the process.

However, the molecular mechanisms underlying this cytoprotective role remain unclear. For further study, we investigated the relationship between mTOR and nestin. Co-IP of the two proteins in podocytes verified the authenticity of the interaction between the two. Our study revealed 
a

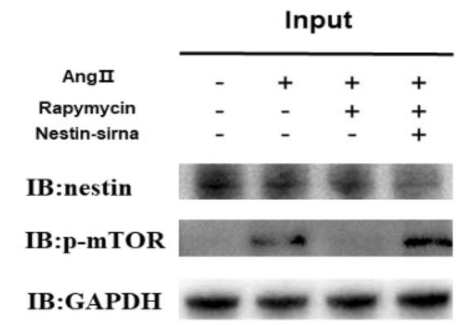

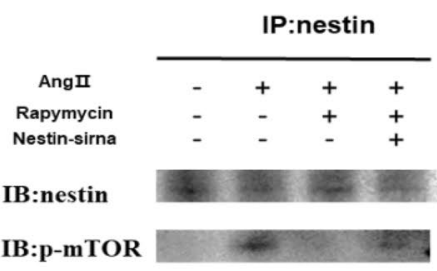

b

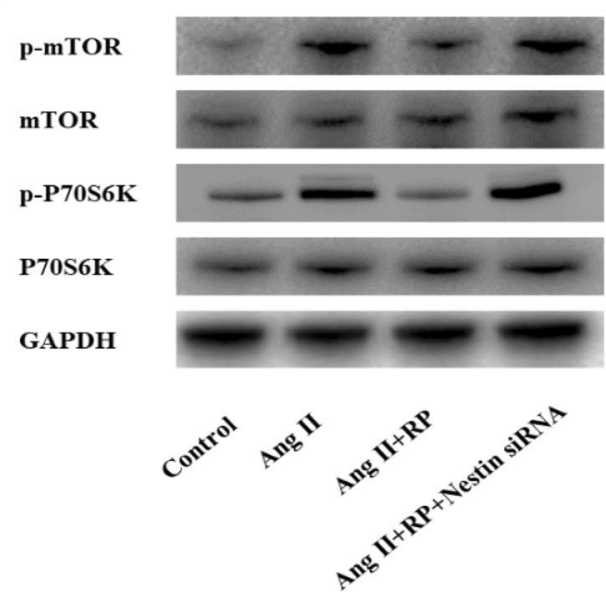

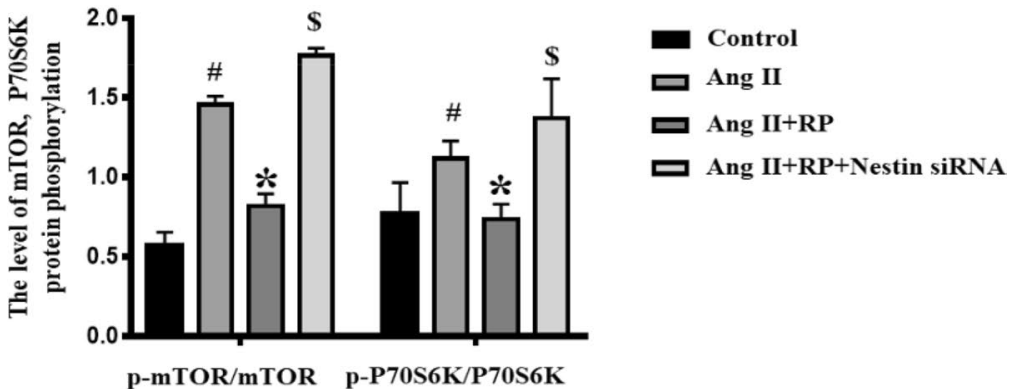

Fig. 4 Nestin combines with p-mTOR and inhibition of nestin enhances mTOR/P70S6K activation. a Co-immunoprecipitation of nestin and p-mTOR in podocytes. Cell lysate was then extracted for co-immunoprecipitation with anti-nestin followed by probing with anti-nestin and anti-p-mTOR after the indicated treatment. b Western

that mTOR interacts with nestin. We then determined the phosphorylation levels of mTOR and P70S6K proteins. We found that nestin affected the protective effect of rapamycin via the mTOR/P70S6K signaling pathway. mTOR is the catalytic subunit of two distinct protein complexes, mTORC1 and mTORC2, which can be distinguished by their unique composition and different substrates [28]. The main function of mTORC1 is the activation of anabolic processes, whereas mTORC2 plays a major role in cytoskeleton organization and cell survival [29]. mTORC1 promotes protein synthesis largely through phosphorylation of p70S6 kinase 1 (P70S6K) effectors. The activation of macroautophagy is a consequence of rapamycin inhibition of mTORC1. Previous findings have indicated that rapamycin disrupts the mTOR-autophagy balance by suppressing the phosphorylation of 4EBP1 and P70S6K in podocytes, resulting in the restoration of podocyte damage [30]. mTORC2 plays an important role in the regulation of cytoskeleton structure [31]. These two mTOR complexes with different functions may cross-talk with each other to balance their signaling. Moreover, either S6K1 or Akt has also been shown to control mTORC2 activity through a recently discovered blotting for mTOR, p-mTOR, P70S6K, and p-P70S6K after the indicated treatment. The data are presented as mean \pm SEM. ${ }^{*} \mathrm{P}<0.05$ vs. control group, $* \mathrm{P}<0.05$ vs. Ang II group. $\$ \mathrm{P}<0.05$ vs. Ang II $+\mathrm{RP}$ group

negative-feedback loop [32]. It was found that mTOR/ P70S6K interacts with Rac1 to recombine with the actin cytoskeleton [33]. However, the underlying mechanism remains unclear and requires further investigation.

In conclusion, our study demonstrates that rapamycin can alleviate Ang II-induced podocyte injury by upregulating the expression of nestin, both in vivo and in vitro. Nestin was found to play a crucial role in podocytes by modulating the mTOR/P70S6K signaling pathway through RNA interference technology and other experiments. This finding not only helps us to elucidate the mechanism of podocyte injury and the protection of rapamycin, but also provides a new potential therapeutic strategy for the treatment of CKD.

Author contributions WG and AZ designed the study. HS and YZ performed the experiments and discussed the results. HS wrote the manuscript. TH, GQ and SL performed the experiments and analyzed the data.

Funding The work was supported by National Natural Science Foundation of China (Grants Nos. 81670650 and 81970664); Jiangsu Province Women and Children Health Key Talents (Grant No. FRC201737); 
Nanjing Health Young Talents (Grant No. QRX17106); 789 Outstanding Talent Program of SAHNMU (Grants Nos. 789ZYRC202080119 and 789ZYRC202090251); The Fund for development of Science and Technology of Nanjing Medical University (Grant No. NMUB2020051).

Data availability The datasets analyzed during the present study are available from the corresponding author on reasonable request.

\section{Declarations}

Conflict of interest The authors declare no conflict of interest.

Ethical approval The study was approved by Institutional Animal Care and Use Committee at Nanjing Medical University.

Open Access This article is licensed under a Creative Commons Attribution 4.0 International License, which permits use, sharing, adaptation, distribution and reproduction in any medium or format, as long as you give appropriate credit to the original author(s) and the source, provide a link to the Creative Commons licence, and indicate if changes were made. The images or other third party material in this article are included in the article's Creative Commons licence, unless indicated otherwise in a credit line to the material. If material is not included in the article's Creative Commons licence and your intended use is not permitted by statutory regulation or exceeds the permitted use, you will need to obtain permission directly from the copyright holder. To view a copy of this licence, visit http://creativecommons.org/licenses/by/4.0/.

\section{References}

1. Nagata M (2016) Podocyte injury and its consequences. Kidney Int 89(6):1221-1230

2. Assady S, Wanner N, Skorecki KL, Huber TB (2017) New insights into podocyte biology in glomerular health and disease. J Am Soc Nephrol 28(6):1707-1715

3. Koppe L, Fouque D (2019) The role for protein restriction in addition to renin-angiotensin-aldosterone system inhibitors in the management of CKD. Am J Kidney Dis 73(2):248-257

4. Ren Z, Liang W, Chen C, Yang H, Singhal PC, Ding G (2012) Angiotensin II induces nephrin dephosphorylation and podocyte injury: role of caveolin-1. Cell Signal 24(2):443-450

5. Yang Y, Yang Q, Yang J, Ma Y, Ding G (2017) Angiotensin II induces cholesterol accumulation and injury in podocytes. Sci Rep $7(1): 10672$

6. Zschiedrich S, Bork T, Liang W, Wanner N, Eulenbruch K, Munder S, Hartleben B, Kretz O, Gerber S, Simons M, Viau A, Burtin M, Wei C, Reiser J, Herbach N, Rastaldi MP, Cohen CD, Tharaux PL, Terzi F, Walz G et al (2017) Targeting mTOR Signaling can prevent the progression of FSGS. J Am Soc Nephrol 28(7):2144-2157

7. Mao J, Zeng Z, Xu Z, Li J, Jiang L, Fang Y, Xu X, Hu Z, He W, Yang J, Dai C (2014) Mammalian target of rapamycin complex 1 activation in podocytes promotes cellular crescent formation. Am J Physiol Renal Physiol 307(9):F1023-F1032

8. Naumovic R, Jovovic D, Basta-Jovanovic G, Miloradovic Z, Mihailovic-Stanojevic N, Aleksic T, Jovanovic D (2007) Effects of rapamycin on active Heymann nephritis. Am J Nephrol 27(4):379-389

9. Zhang HT, Wang WW, Ren LH, Zhao XX, Wang ZH, Zhuang DL, Bai YN (2016) The mTORC2/Akt/NFkB pathway-mediated activation of TRPC6 participates in adriamycin-induced podocyte apoptosis. Cell Physiol Biochem Int J Exp Cell Physiol Biochem Pharmacol 40(5):1079-1093

10. Thomson AW, Turnquist HR, Raimondi G (2009) Immunoregulatory functions of mTOR inhibition. Nat Rev Immunol 9(5):324-337

11. Li J, Kim SG, Blenis J (2014) Rapamycin: one drug, many effects. Cell Metab 19(3):373-379

12. Yu R, Bo H, Villani V, Spencer PJ, Fu P (2016) the inhibitory effect of rapamycin on toll like receptor 4 and interleukin 17 in the early stage of rat diabetic nephropathy. Kidney Blood Press Res 41(1):55-69

13. Lendahl U, Zimmerman LB, McKay RD (1990) CNS stem cells express a new class of intermediate filament protein. Cell 60(4):585-595

14. Chen J, Boyle S, Zhao M, Su W, Takahashi K, Davis L, Decaestecker M, Takahashi T, Breyer MD, Hao CM (2006) Differential expression of the intermediate filament protein nestin during renal development and its localization in adult podocytes. J Am Soc Nephrol 17(5):1283-1291

15. Tian Y, Guo H, Miao X, Xu J, Yang R, Zhao L, Liu J, Yang L, Gao F, Zhang W, Liu Q, Sun S, Tian Y, Li H, Huang J, Gu C, Liu S, Feng X (2020) Nestin protects podocyte from injury in lupus nephritis by mitophagy and oxidative stress. Cell Death Dis 11(5):319

16. Huang H, Fan Y, Gao Z, Wang W, Shao N, Zhang L, Yang Y, Zhu W, Chen Z, Hu J, Ding G (2019) HIF-1 $\alpha$ contributes to Ang II-induced inflammatory cytokine production in podocytes. BMC Pharmacol Toxicol 20(1):59

17. Ji J, Zhao Y, Na C, Yang M, Zhu X, Shi H, Gan W, Zhang A (2019) Connexin 43-autophagy loop in the podocyte injury of diabetic nephropathy. Int J Mol Med 44(5):1781-1788

18. Yadav A, Vallabu S, Arora S, Tandon P, Slahan D, Teichberg S, Singhal PC (2010) ANG II promotes autophagy in podocytes. Am J Physiol Cell Physiol 299(2):C488-C496

19. Gao Z, Chen X, Zhu K, Zeng P, Ding G (2017) Dab1 contributes to angiotensin II-induced apoptosis via p38 signaling pathway in podocytes. Biomed Res Int 2017:2484303

20. Saxton RA, Sabatini DM (2017) mTOR signaling in growth, metabolism, and disease. Cell 169(2):361-371

21. Puelles VG, van der Wolde JW, Wanner N, Scheppach MW, Cullen-McEwen LA, Bork T, Lindenmeyer MT, Gernhold L, Wong MN, Braun F, Cohen CD, Kett MM, Kuppe C, Kramann R, Saritas T, van Roeyen CR, Moeller MJ, Tribolet L, Rebello R, Sun YB et al (2019) mTOR-mediated podocyte hypertrophy regulates glomerular integrity in mice and humans. JCI Insight 4(18):e99271

22. Gödel M, Hartleben B, Herbach N, Liu S, Zschiedrich S, Lu S, Debreczeni-Mór A, Lindenmeyer MT, Rastaldi MP, Hartleben G, Wiech T, Fornoni A, Nelson RG, Kretzler M, Wanke R, Pavenstädt H, Kerjaschki D, Cohen CD, Hall MN, Rüegg MA et al (2011) Role of mTOR in podocyte function and diabetic nephropathy in humans and mice. J Clin Investig 121(6):2197-2209

23. Ma H, Togawa A, Soda K, Zhang J, Lee S, Ma M, Yu Z, Ardito T, Czyzyk J, Diggs L, Joly D, Hatakeyama S, Kawahara E, Holzman L, Guan JL, Ishibe S (2010) Inhibition of podocyte FAK protects against proteinuria and foot process effacement. J Am Soc Nephrol 21(7):1145-1156

24. Lieberthal W, Levine JS (2009) The role of the mammalian target of rapamycin (mTOR) in renal disease. J Am Soc Nephrol 20(12):2493-2502

25. Liu W, Zhang Y, Hao J, Liu S, Liu Q, Zhao S, Shi Y, Duan H (2012) Nestin protects mouse podocytes against high glucoseinduced apoptosis by a Cdk5-dependent mechanism. J Cell Biochem 113(10):3186-3196

26. Mathieson PW (2012) The podocyte cytoskeleton in health and in disease. Clin Kidney J 5(6):498-501 
27. Sunabori T, Tokunaga A, Nagai T, Sawamoto K, Okabe M, Miyawaki A, Matsuzaki Y, Miyata T, Okano H (2008) Cellcycle-specific nestin expression coordinates with morphological changes in embryonic cortical neural progenitors. J Cell Sci 121(Pt 8):1204-1212

28. Jhanwar-Uniyal M, Wainwright JV, Mohan AL, Tobias ME, Murali R, Gandhi CD, Schmidt MH (2019) Diverse signaling mechanisms of mTOR complexes: mTORC1 and mTORC2 in forming a formidable relationship. Adv Biol Regul 72:51-62

29. Kazyken D, Magnuson B, Bodur C, Acosta-Jaquez HA, Zhang D, Tong X, Barnes TM, Steinl GK, Patterson NE, Altheim CH, Sharma N, Inoki K, Cartee GD, Bridges D, Yin L, Riddle SM, Fingar DC (2019) AMPK directly activates mTORC2 to promote cell survival during acute energetic stress. Sci Signal 12(585):eaav3249

30. Jin J, Hu K, Ye M, Wu D, He Q (2018) Rapamycin reduces podocyte apoptosis and is involved in autophagy and mTOR/ P70S6K/4EBP1 signaling. Cell Physiol Biochem Int J Exp Cell Physiol Biochem Pharmacol 48(2):765-772
31. Lamming DW, Ye L, Katajisto P, Goncalves MD, Saitoh M, Stevens DM, Davis JG, Salmon AB, Richardson A, Ahima RS, Guertin DA, Sabatini DM, Baur JA (2012) Rapamycin-induced insulin resistance is mediated by mTORC2 loss and uncoupled from longevity. Science (New York, N.Y.) 335(6076):1638-1643

32. Zou Z, Chen J, Yang J, Bai X (2016) Targeted inhibition of Rictor/ mTORC2 in cancer treatment: a new era after rapamycin. Curr Cancer Drug Targets 16(4):288-304

33. Aslan JE, Tormoen GW, Loren CP et al (2011) S6K1 and mTOR regulate Rac1-driven platelet activation and aggregation. Blood 118(11):3129-3136

Publisher's Note Springer Nature remains neutral with regard to jurisdictional claims in published maps and institutional affiliations. 